\title{
Edge Enhancement Algorithm Based on the Wavelet Transform for Automatic Edge Detection in SAR Images
}

\author{
Mariví Tello Alonso, Student Member, IEEE, Carlos López-Martínez, Member, IEEE, \\ Jordi J. Mallorquí, Member, IEEE, and Philippe Salembier, Senior Member, IEEE
}

\begin{abstract}
This paper presents a novel technique for automatic edge enhancement and detection in synthetic aperture radar (SAR) images. The characteristics of SAR images justify the importance of an edge enhancement step prior to edge detection. Therefore, this paper presents a robust and unsupervised edge enhancement algorithm based on a combination of wavelet coefficients at different scales. The performance of the method is first tested on simulated images. Then, in order to complete the automatic detection chain, among the different options for the decision stage, the use of geodesic active contour is proposed. The second part of this paper suggests the extraction of the coastline in SAR images as a particular case of edge detection. Hence, after highlighting its practical interest, the technique that is theoretically presented in the first part of this paper is applied to real scenarios. Finally, the chances of its operational capability are assessed.
\end{abstract}

Index Terms-Edge detection, geodesic active contour, synthetic aperture radar (SAR), wavelet transform.

\section{INTRODUCTION}

$\mathbf{S}$ ATELLITE-BORNE synthetic aperture radars (SARs) allow the observation of broad expanses during the day as well as during the night and as independent from weather effects. These characteristics, added to a high-resolution capability, make them particularly helpful for the global observation of the Earth for environmental and security issues. Nevertheless, at first sight, a SAR image which represents the complex reflectivity map of a scene is not meaningful for an inexperienced observer since, unlike optical images, the interpretation of the radar images is not consistent with a common visual perception. Furthermore, the direct application of conventional image processing tools, conceived from an optical point of view, usually gives suboptimum results on SAR data. Hence, specific data analysis algorithms are still to be provided in order

Manuscript received October 7, 2009; revised March 13, 2010; accepted May 21, 2010. Date of publication July 26, 2010; date of current version December 27, 2010. This work was supported in part by the Spanish MICINN and FEDER funds under Project TEC2008-06764-C02-01 and in part by the Ramon y Cajal Program.

M. Tello Alonso, C. López-Martínez, and J. J. Mallorquí are with the Remote Sensing Laboratory, Department of Signal Theory and Communications (TSC), Universitat Politècnica de Catalunya (UPC), 08034 Barcelona, Spain (e-mail: carlos.lopez@tsc.upc.edu).

P. Salembier is with the Image and Video Processing Group, Department of Signal Theory and Communications (TSC), Universitat Politècnica de Catalunya (UPC), 08034 Barcelona, Spain.

Color versions of one or more of the figures in this paper are available online at http://ieeexplore.ieee.org.

Digital Object Identifier 10.1109/TGRS.2010.2052814 to assure unsupervised and robust means for the intensive and operational exploitation of SAR data.

Robust edge detection techniques are essentially based on the following two steps: edge enhancement and decision. Unlike optical images, in SAR data, which is highly heterogeneous, a robust edge enhancement phase is critical in providing acceptable detection rates. This phase is usually performed through techniques that are related to derivation, namely, simple differences, Sobel filter [1], Prewitt filter [2], morphological gradients, etc., possibly combined with smoothing. These methods provide a limited efficiency in SAR applications due to the presence of a speckle which is a multiplicative noiselike pattern [3]. This paper aims at presenting a novel method for edge enhancement in SAR images based on the exploitation of the information provided by the wavelet coefficients.

In the past, several multiscale approaches, relying on the analysis of the information contained in the wavelet domain, have been proposed [4]-[7]. The method proposed in this paper is based on a different way of managing multiscale data. Moreover, it does not assume any statistical distribution of the input data nor any particular type of edge, and it works exclusively in the transformed domain. Further differences of operation between these methods and the proposed technique will be highlighted throughout the paper. In the scope of SAR images, the study in [8] proposes an interesting review of existing edge detection algorithms. A group of techniques is based on the evaluation of the ratio of averages over a sliding window [9]-[12]. These methods present a low computational load, but they are highly dependent on the dimensions of the window and are not robust in noisy scenes. Several algorithms are based on the wavelet theory. For instance, the study in [13] or [14] proposes an edge detector based on a threshold operation of wavelet coefficients. Despite a low computational cost and a good contrast, detected edges are too thick. The approach proposed in this paper will tackle at the same time the robustness and the precision issues of edge enhancement and detection.

In Section II, we will discuss the overview of the characteristics of SAR images and especially those that make their processing through conventional methods inefficient. Then, the use of a multiscale framework will be justified in Section III. The multiscale algorithm proposed for edge enhancement in SAR images will be presented in Section IV. The chain for an unsupervised edge detection will be completed with a geodesic 


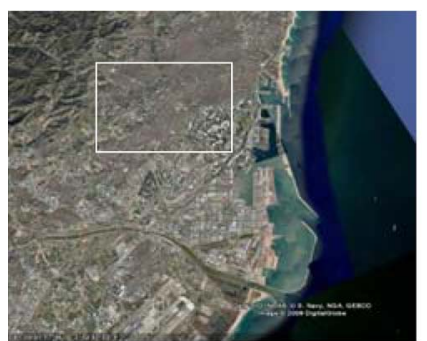

(a)

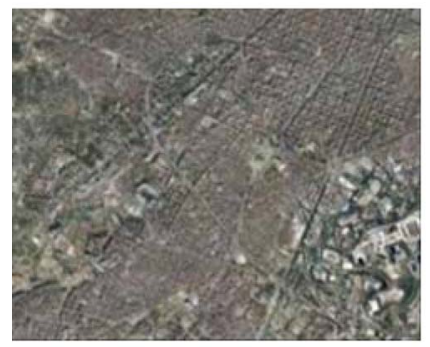

(c)

Fig. 1. Same coastal urban scene (Barcelona, Spain) as observed (a) by an optical spaceborne sensor (source: GoogleEarth) and (b) by SAR (source: ERS). (c) and (d) show the zoomed image of a small area.

active contour technique [30] in Section V. Finally, Section VI will draw the conclusion of this paper.

\section{SAR IMAGES}

Complex radar data are proportional to the scattering properties of the observed scene at the corresponding microwave frequency. Thus, a radar image consists of a mapping of electromagnetic measurements, characterized by a large dynamic range that may even reach $40 \mathrm{dBs}$. Hence, it does not correspond to a representation that is fully comprehensible through a common visual perception. The image in Fig. 1(a) has been acquired by the QuickBird optical spaceborne sensor. A human observer, assisted by his experience of aerial pictures, may easily identify a coastal urban scenario, clearly distinguishing streets and buildings. Moreover, this interpretation is valid when confronted of the overall scene, and it persists when zooming into smaller fragments of it [see Fig. 1(c)]. In contrast, the SAR intensity image [Fig. 1(b)] represents the same coastal scenario, as imaged by a spaceborne SAR sensor that is onboard the ERS-1 satellite. This type of data is hardly attractive for an inexperienced user even if a specialist would be able to discriminate not only the presence of buildings, streets, and squares but also additional information such as, for example, quarters occupied by higher buildings or a rough qualitative estimation of a wind field in the sea surface. In addition, the SAR images are even less explicit when considering a small portion of them [see the detailed image in Fig. 1(d)] as, at small scales, the SAR images reveal the presence of the speckle noise [15].

Specifically, a complex SAR image may be represented as the convolution of the local complex reflectivity of the observed area $\gamma(\tau, \eta)$ with the impulse response of the SAR system $u_{0}(\tau, \eta)[15]$

$$
u(\tau, \eta)=\gamma(\tau, \eta) * u_{0}(\tau, \eta)
$$

where $*$ stands for the convolution and $\tau$ and $\eta$ are the azimuth and the range coordinates, respectively, employed to define the coordinate system of a SAR image. The SAR system impulse response $u_{0}(\tau, \eta)$ may be interpreted as a bidimensional lowpass filter [16], in such a way that the finite local support of this filter determines the spatial resolution of the SAR system. The spatial area embraced by this impulse response is known as the resolution cell. Hence, the SAR data pixels are the lowpass-filtered version of the complex local scattering properties of the observed scene. These values may be quantitatively interpreted, considering the use of electromagnetic scattering models, making possible the retrieval of geo- and biophysical information [17], [18].

The SAR imaging process indicated by (1) admits a simple mathematical model that considers linearity and Born approximation, i.e., the imaged scene is modeled as a set of $N$ individual scatterers whose scattered fields superpose linearly [19]

$$
u(\tau, \eta)=\sum_{k=1}^{N} \gamma_{k}(\tau, \eta) * u_{0}(\tau, \eta)
$$

where $\gamma_{k}(\tau, \eta)$ is the complex reflectivity of the $k$ th individual scatterer within the resolution cell. The total imaged signal may also be rewritten for an easier interpretation

$$
u(\tau, \eta)=\sum_{k=1}^{N} a_{k} e^{j \phi_{k}}
$$

where $a_{k}$ and $\phi_{k}$ are the amplitude and the phase of the contribution of the $k$ th scatterer. The interpretation of (3) must be done according to $N$. When the resolution cell presents a single individual scatterer or when the total returned wave is dominated by one individual scatterer, the pixel is said to represent a point or deterministic target. In this situation, the complex value of the pixel must be interpreted in terms of the physical properties and geometry of the imaged scatterer [20]. When the number of scatterers $N$ increases, the complex value of the pixel results from the coherent addition of $N$ complex quantities that may be interpreted as a bidimensional random walk process. When $N$ is low, the characterization of $u(\tau, \eta)$ is complex [21]. Nevertheless, if $N$ is large enough, assuming that the individual scatterers are independent, that $\left|a_{k}\right|$ and $\phi_{k}$ are also unrelated, and that the phases $\phi_{k}$ are uniformly distributed in $[-\pi, \pi)$, according to the central limit theorem, it may be proved that the real and imaginary parts $u(\tau, \eta)$ may be modeled as independent, zero-mean, and equally distributed Gaussian random variables [3]. At this stage, the classical random walk problem in the complex plane becomes complete. Consequently, the intensity of a SAR image (i.e., $\left.I=|u(\tau, \eta)|^{2}\right)$ follows a negative exponential distribution

$$
p_{I}(I)= \begin{cases}\frac{1}{\sigma} e^{-\frac{I}{\sigma}}, & I \geq 0 \\ 0 & \text { otherwise }\end{cases}
$$

with the mean value and standard deviation both equal to $\sigma$. The phase of $u(\tau, \eta)$ is uniformly distributed in $[-\pi, \pi)$, and hence, it contains no information concerning the imaged scene. Under these hypotheses, the imaged target is said to be distributed, where the value of a given pixel $u(\tau, \eta)$ represents a true 


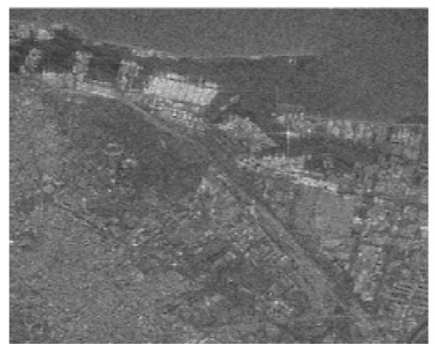

(a)

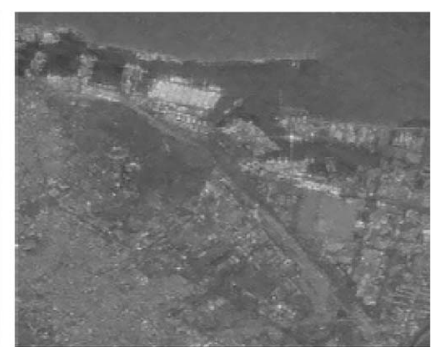

(b)

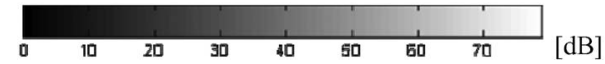

Fig. 2. Example of the application of the Lee filter with a window size of $11 x$ 11 pixels in a SAR image of the area of Barcelona. (a) Detail of a SAR image. (b) Result of the Lee filter.

electromagnetic measurement. Nevertheless, the complexity of (3) makes it only possible to interpret the value of the pixel in statistical terms, characterizing it by its mean value $\sigma$. Consequently, the intensity $I(\tau, \eta)$ may be written as

$$
I=\sigma n \text {. }
$$

In the following, the dependence on $\tau$ and $\eta$ shall be assumed. The useful information is determined by the parameter $\sigma$, as it represents the radar cross section of the scene that is normalized to the resolution cell area [15], whereas $n$ is a multiplicative noise component characterized by a negative exponential distribution with a mean value that is equal to one (i.e., $p_{n}(n)=e^{-n}$ ). The term $n$ is referred to as speckle. Despite being a true electromagnetic measurement, the speckle must be considered as a noise component due to the complexity associated with the scattering process.

The speckle represents one of the major drawbacks in SAR image interpretation. Consequently, this component is usually filtered in order to grant access to the useful information. In terms of SAR image postprocessing, the speckle leads to over segmentations, and filters are often employed to reduce its effects. However, even if they are able to effectively smooth the speckle, they inevitably affect the information, and its application results in a degradation of the spatial resolution. Among the speckle filters proposed in the literature, the most relevant ones are the Lee filter [22], the Kuan filter [23], the gamma filter [24], and the Frost filter [25]. In the scope of this paper, the Lee filter will be employed later on for comparison purposes. Fig. 2 shows the effect of Lee filtering through an example of the application over a SAR image of a coastal area.

\section{Multiscale FrameWORK FOR THE EXPLOITATION OF SAR DATA}

\section{A. SAR Data Product Model}

A multiscale framework is proposed for the exploitation of SAR data since SAR images present themselves a multiscale nature. For instance, as shown in the previous section, the speckle is produced by the combination of scatterers within a resolution cell, and thus, it can be considered as a pixelto-pixel, equivalently a small scale, spatial variability characteristic. Moreover, a multiplicative model for the speckle has been presented in (5). Nevertheless, the validity of this model is restricted to homogeneous areas and results that are insufficient in describing more complex scenarios that are present in true SAR data as, for instance, textured areas. In these cases, it is necessary to increase the complexity of the statistical model describing the imaged area: variations that correspond to an intrinsic texture in a nonhomogeneous area tend to be appreciable at larger scales. The product model [26] formalizes this multiresolution notion stating intensity $I$

$$
I=\mu_{I} T F
$$

where $\mu_{I}$ is the mean intensity, $T$ is the texture random variable and the representation of the natural within-field spatial variability, and $F$ corresponds to the speckle which is also a random process. $T$ and $F$ are considered statistically independent. This decomposition enables the identification of different ranges of scales. As a consequence, in order to analyze properly the SAR data, the consideration of a multiscale approach is the most suitable.

This multiscale notion can be translated intuitively by considering the example that is shown in Fig. 1. The observation of a small area of pixels of a SAR image [Fig. 1(d)] exhibits a noiselike pattern that is meaningless in appearance. Nevertheless, even if the speckle affects all scales due to its multiplicative nature, when confronted by a larger scene [see, for example, Fig. 1(b)], an observer is able to manage it and is able to distinguish the most relevant features, focusing its multiscale observation capability to higher scales, i.e., lower frequencies or larger areas. In Fig. 1(b), a coastal scene with different characteristic elements (the sea, the land, the harbor, and an urban area) can easily be discriminated.

\section{B. Multiscale Analysis by Means of the Wavelet Transform}

Among the different tools of multiscale signal processing, this paper is focused on time-frequency analysis with wavelets [27]. For the purpose of edge enhancement, it is useful to interpret the wavelet transform as a multiscale differential operator. More specifically, if a wavelet $\psi$ has a compact support and $n$ vanishing moments, i.e.,

$$
\int_{-\infty}^{+\infty} t^{k} \psi(t) d t=0, \quad \text { for } 0 \leq k<n
$$

there exists a function $\theta$ with a fast decay such that

$$
\psi(t)=(-1)^{n} \frac{d^{n} \theta(t)}{d t^{n}} .
$$

Then, the wavelet transform of a signal $f$ can be expressed as

$$
W f(u, s)=s^{n} \frac{d^{n}}{d u^{n}}\left(f * \bar{\theta}_{s}\right)(u)
$$

where $\bar{\theta}_{s}(t)=s^{-1 / 2} \theta(-t / s), u$ is the time or space coordinate, and $s$ is the scale. As a consequence, under these conditions, the wavelet transform $W f(u, s)$ is an $n$ th-order derivative of an averaging of $f$, with $\bar{\theta}_{s}$ over a domain proportional to $s$. 
When using wavelet tools for signal processing purposes, it is critical to choose conveniently the type of transform as well as the mother wavelet according to the nature of the signal to be analyzed and according to the type of characteristic to be highlighted. On the one hand, in the framework of this paper, a discrete stationary wavelet transform (SWT) will be employed. It is an inherently redundant scheme that is similar to the discrete wavelet transform without subsampling [27]. As a consequence, if using a 1-D wavelet transform, two frequency bands are obtained at each iteration. In the case of a 2-D transform, it is four subbands. The first three bands (i.e., $\{H, V, D\})$ refer to the horizontal, vertical, and diagonal details of the image, respectively. The fourth band contains the low-pass-filtered component of the image. In both cases, each component has the same size as that of the input signal. For instance, the algorithm for edge enhancement that is proposed in this paper is based on the combination of wavelet coefficients at different scales, and it is thus necessary for the coefficients to be combined to generate an image of the same size as the input image. This justifies the choice of the SWT.

On the other hand, in order to select the appropriate mother wavelet, the size of its support both in time and in frequency, as well as its number of vanishing moments, has to be taken into account. In the application concerning this paper, it is important to preserve as precisely as possible the location of the edge, and hence, a short spatial support is preferred. Furthermore, it has been seen previously that SAR images tend to be irregular, presenting a lot of discontinuities due to the multiplicative nature of the speckle. As a consequence, a large number of vanishing moments are not necessary for their analysis. Moreover, the proposed algorithm relies on enhancing edges, taking benefit of the spatial coincidence of the local maxima at different scales due to the presence of discontinuities: the maxima produced by the presence of a frontier tend to persist over scales, resulting in a higher interscale spatial correlation in the presence of an edge than in the background [27]. In order to effectively have this spatial co-occurrence, the mother wavelet used must exhibit a linear phase and a small number of coefficients. For all of these reasons, the Haar wavelet with two coefficients, a single vanishing moment, and a linear phase has been selected for the addressed application.

\section{Edge Enhancement Algorithm}

\section{A. Theoretical Principles}

The algorithm for edge enhancement in SAR images, which is proposed in this paper, relies on the difference of behavior along the wavelet scales of the speckle in front of the edges. On the one hand, the discontinuities are highlighted by the wavelet transform, and they tend to persist over scales (see Fig. 3).

On the other hand, the speckle is progressively smoothen, and moreover, it is almost spatially uncorrelated between scales (see Fig. 4).

The wavelet transform can be expressed as

$$
W f(u, s)=\int_{-\infty}^{\infty} f(x) \frac{1}{\sqrt{s}} \psi^{*}\left(\frac{x-u}{s}\right) d x
$$

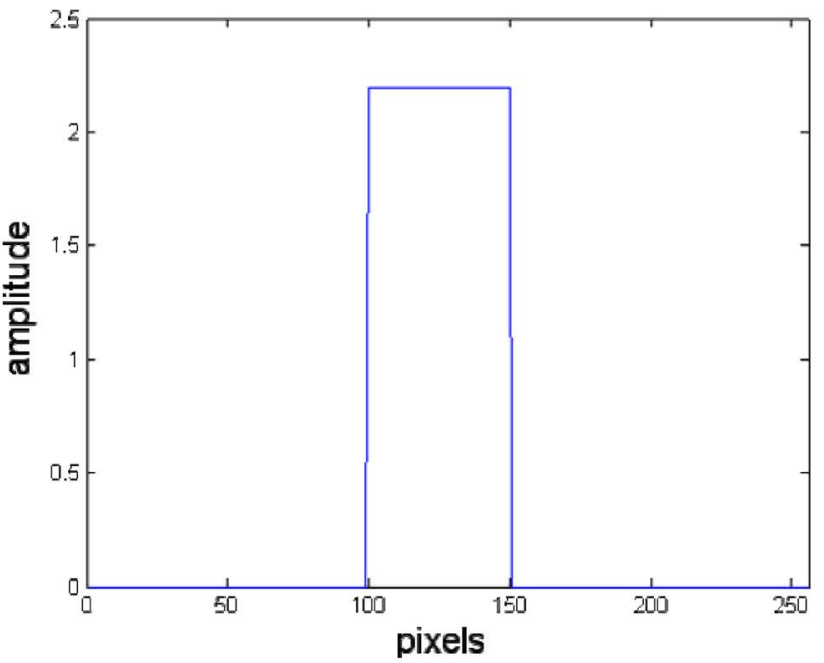

(a)

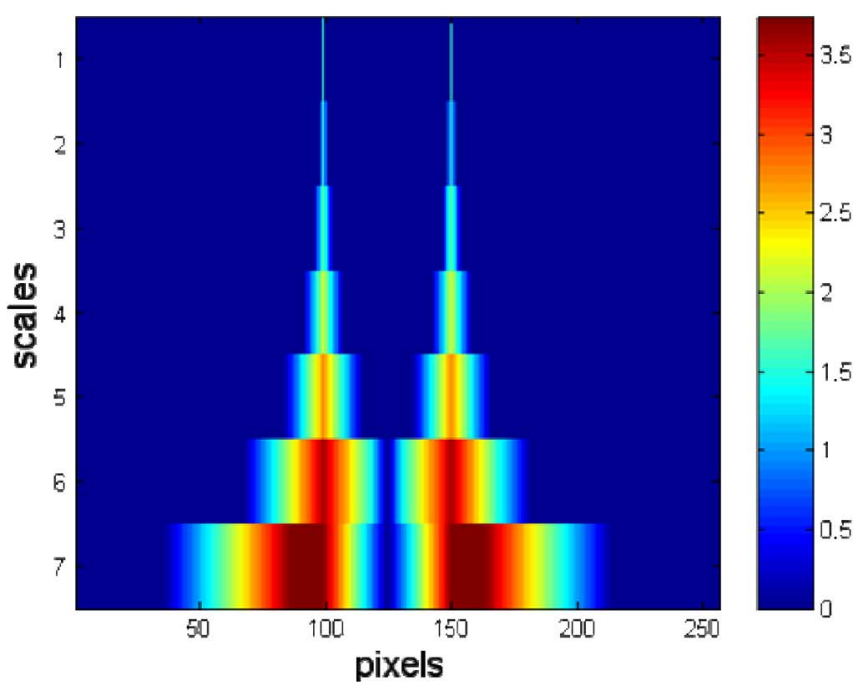

(b)

Fig. 3. (a) Simulated step signal and (b) its scale/space representation, obtained through the SWT with a Haar mother wavelet.

The Haar wavelet can be expressed as

$$
\psi(t)= \begin{cases}1, & 0 \leq t<\frac{1}{2} \\ -1, & \frac{1}{2} \leq t<1 \\ 0, & \text { otherwise }\end{cases}
$$

With this

$$
W f(u, s)=\frac{1}{\sqrt{s}} \int_{u}^{s / 2+u} f(x) d x-\frac{1}{\sqrt{s}} \int_{s / 2+u}^{s+u} f(x) d x .
$$

In the framework of SAR processing, it is useful to take the logarithm of the original signal in order to manage the multiplicative speckle [28]. Then, we can suppose

$$
f(\tau)=\log (\sigma(\tau) n(\tau))=\log (\sigma(\tau))+\log (n(\tau))
$$

where $n$ stands for the speckle and $\sigma$ stands for the useful information content of the radar signal. The speckle that is transformed in this way is not only additive and signal independent, but its probability density distribution is also approximately 


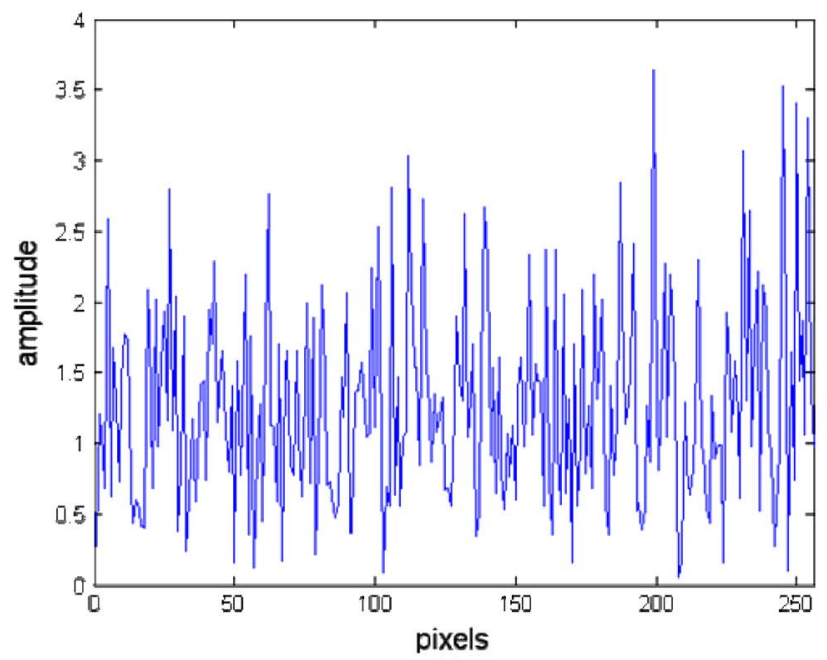

(a)

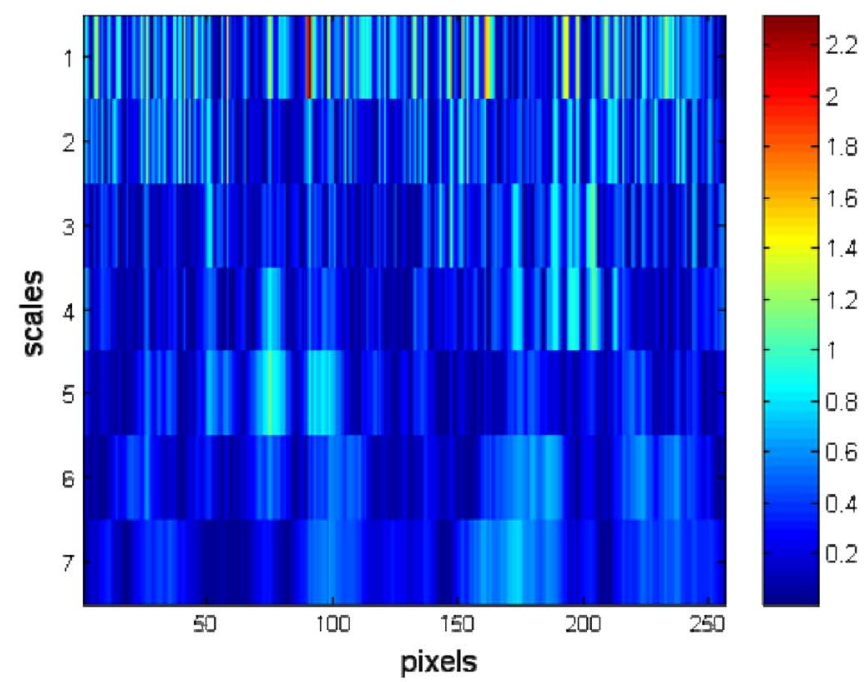

(b)

Fig. 4. (a) Simulated 1-D speckle signal and (b) its scale/space representation, obtained through the SWT with a Haar mother wavelet.

Gaussian [28]. Moreover, the logarithm operation is helpful in reducing the large dynamic range of SAR data. With this, (12) turns into

$$
\begin{aligned}
W f(u, s)= & \frac{1}{\sqrt{s}} \int_{u}^{s / 2+u}[\log (\sigma(x))+\log (n(x))] d x \\
& -\frac{1}{\sqrt{s}} \int_{s / 2+u}^{s+u}[\log (\sigma(x))+\log (n(x))] d x \\
W f(u, s)= & \frac{1}{\sqrt{s}}\left[\int_{u}^{s / 2+u} \log (\sigma(x)) d x-\int_{s / 2+u}^{s+u} \log (\sigma(x)) d x\right] \\
& +\frac{1}{\sqrt{s}}\left[\int_{u}^{s / 2+u} \log (n(x)) d x\right. \\
& \left.-\int_{s / 2+u}^{s+u} \log (n(x)) d x\right] .
\end{aligned}
$$

As a consequence, the wavelet transform may be seen as the difference between the averaging of the curves over two adjacent intervals of the same duration. If the function is homogeneous along both intervals, the absolute value of the difference will be low. On the contrary, if there is a discontinuity in the trend of the curve, it will increase. Moreover, the maximum of this difference occurs when the discontinuity happens just in the frontier between the intervals. Hence, in the wavelet transform of the logarithm of the SAR signal, the influence of the speckle is low since its contribution in each of the semiintervals counteracts with the other. More specifically, if we assume the homogeneity of the speckle

$$
\int_{u}^{s / 2+u} \log (n(x)) d x \approx \int_{s / 2+u}^{s+u} \log (n(x)) d x .
$$

Hence

$$
\int_{u}^{s / 2+u} \log (n(x)) d x-\int_{s / 2+u}^{s+u} \log (n(x)) d x \approx 0 .
$$

It is worth noting that this value is especially low when the interval is large since it permits having a sample that sufficiently represents the stochastic speckle process. On the other hand, the absolute value of the transform accounts for the difference of homogeneity of the useful part of the speckle-free signal between both subintervals.

When considering a single scale of the wavelet transform, it may be observed in (15) that the size of the interval, directly determined by the scale, influences the capability of the wavelet transform both to localize the precise position of the edge and to be unaffected by the speckle. For a large interval size, the effect of the averaging is more important, and the presence of the speckle is, thus, better cancelled through the counteraction of both integrals. Nevertheless, in that case, the higher sensitivity in the detection of the discontinuity is obtained at the expense of a lower precision in the estimation of its location. Since this tradeoff is inherent to a time-frequency decomposition, it is unavoidable if a single scale is taken into account (see Fig. 5).

We propose, instead, a combination of several scales in order to take benefit of the good performance in the resolution of the lower ones and in order to take benefit of the sensitivity to the presence of discontinuities of the higher ones. The combination is performed through an interscale pointwise product which permits taking advantage simultaneously of the benefits of the span of the considered scales. Low values of the speckle that are achieved with intervals of large lengths are reduced through the multiplication of the higher values present in higher scales. Moreover, due to their spatial co-occurrence, the local maxima that are due to a discontinuity contribute constructively when multiplying the scales. Since the energy that is due to the irregularity of the signal is mainly located at low scales, the resolution of the fine scales is preserved. Furthermore, while discontinuities that are due to the speckle do not persist, the ones produced by the presence of a meaningful edge propagate 


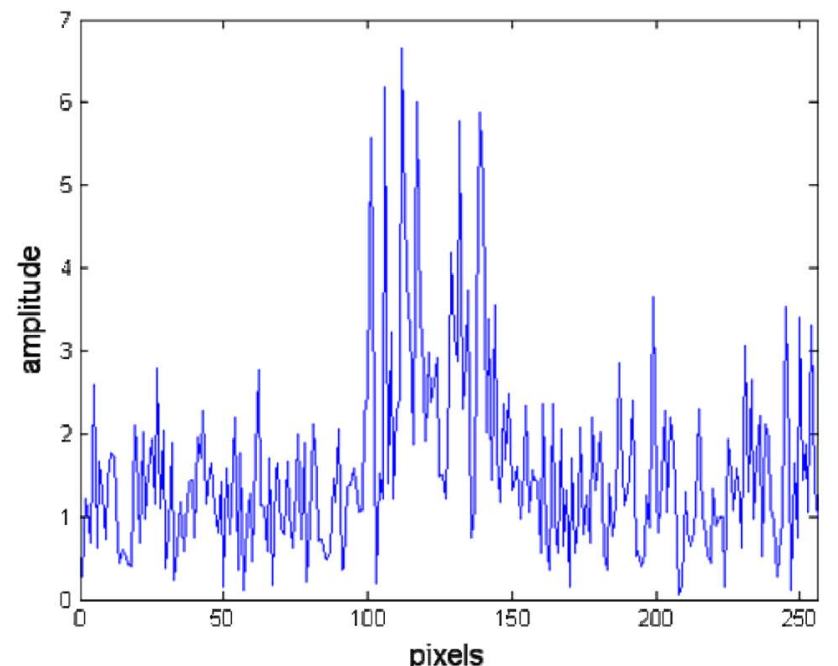

(a)

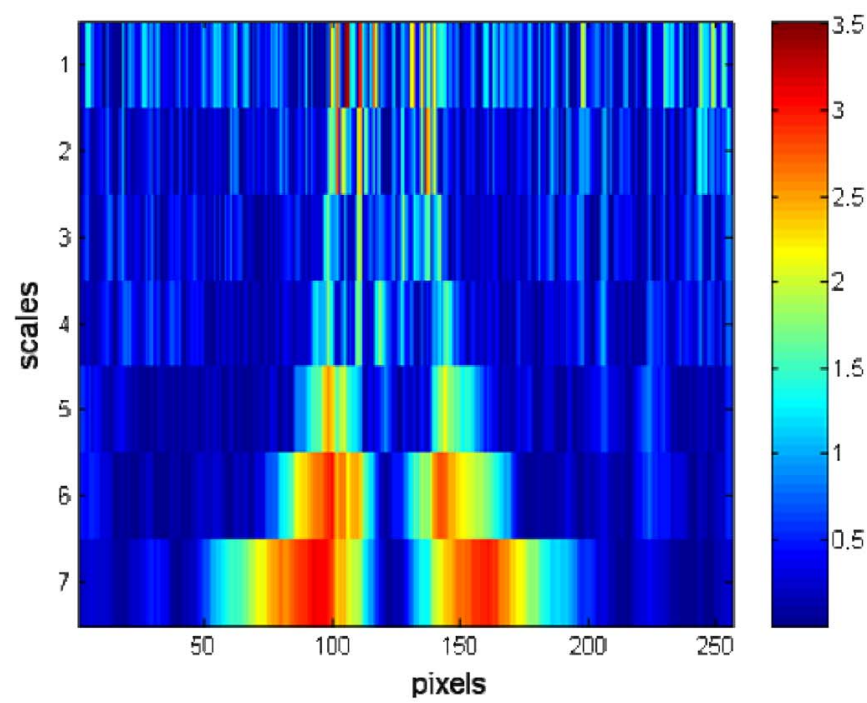

(b)

Fig. 5. (a) Logarithm transform of a simulated 1-D step signal and (b) its scale/space representation, obtained through the SWT with a Haar mother wavelet.

over the scales. As a consequence, the interscale pointwise product neglects the small isolated discontinuities. In [4], the statistical characteristics of the multiscale product are analyzed. It is shown that the additive Gaussian noise is uncorrelated between scales and that the resulting distribution of probabilities is generally non-Gaussian heavy tailed.

Based on these observations, we propose the algorithm that is shown in Fig. 6. At each iteration of the SWT in two dimensions, three bandpass components are obtained, where each one enhances the discontinuities in a different direction. After normalizing each of these subbands to their maximum and taking their absolute value, the pointwise maximum across all of the three subbands is evaluated. A correct implementation of the algorithm proposed here must also deal with situations where a subband does not contain a vessel, as the proposed normalization may lead to noise amplification. In order to deal with this effect, an improved normalization step performs first an evaluation of the relative level of the noise floor with respect to the maximum in each wavelet subband. If a feature (i.e.,

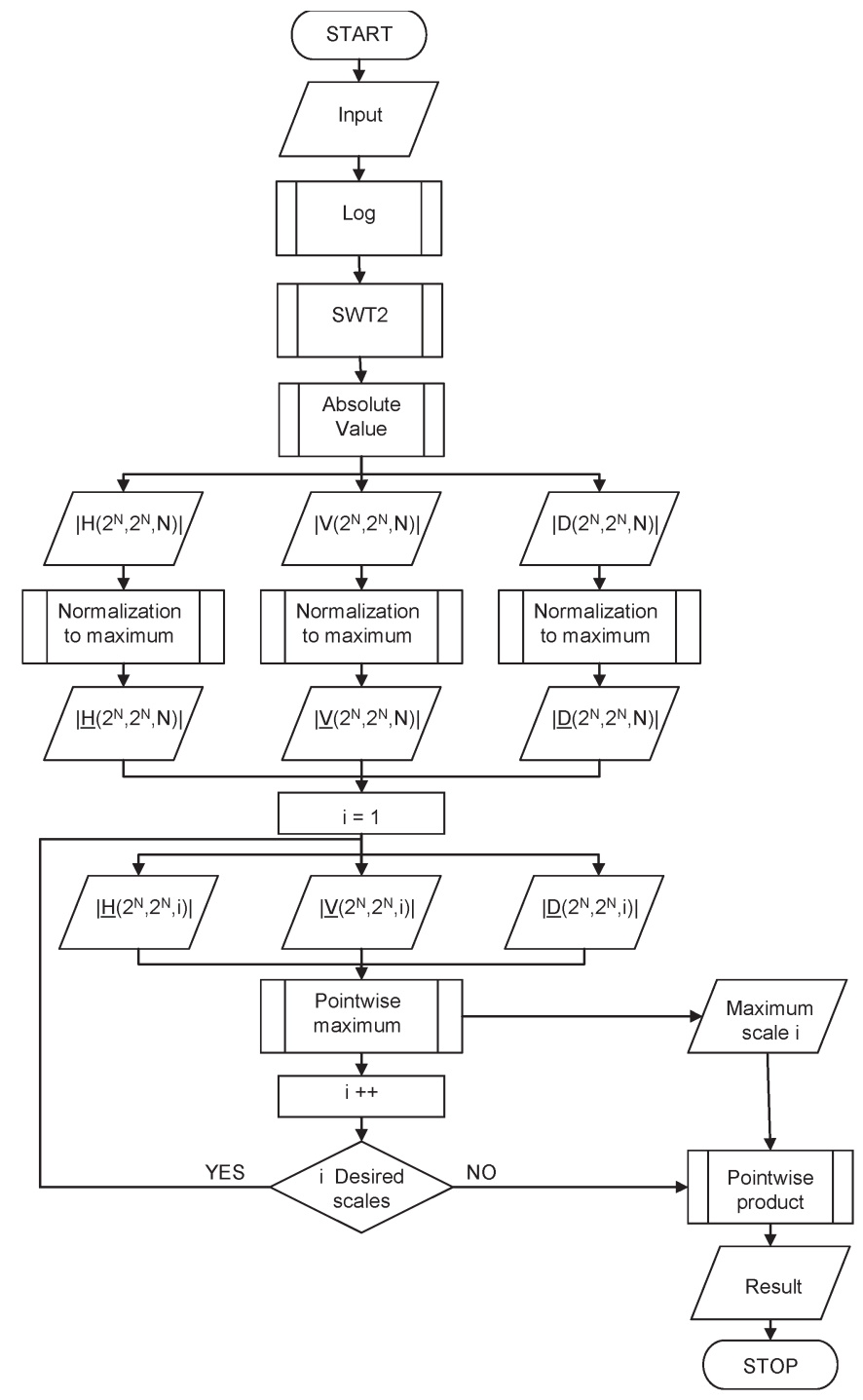

Fig. 6. Flowchart of the proposed algorithm for edge enhancement.

a vessel) is present in a particular subband, the histogram shall be heavy tailed, and the normalization is performed as usual using the maximum of the corresponding subband. If not, the normalization factor is a number of times (usually three) the standard deviation in order to ensure low values on this subband. Since, the components in the same scale are uncorrelated, taking the maximum value pixel per pixel permits avoiding as much as possible the speckle, which is contrary to an intrascale combination with the sum such as in [6]. The same operation is carried out with different scales. Then, the different intermediate maxima previously calculated are combined through pointwise multiplication: For each pixel, the output value corresponds to the product of the values for that pixel of the intermediate maxima matrices obtained in the previous step.

If necessary and if some kind of a priori information is available, the number of iterations can be adjusted accordingly. Otherwise, in common situations, five iterations suffice to provide satisfactory results regarding the usual range of the resolutions of SAR images. 

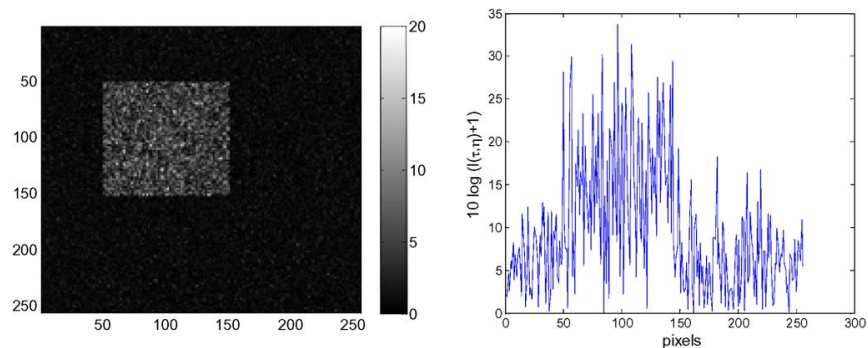

Fig. 7. Logarithm transform of a simulated image constituted by a square embedded in (left) a speckle matrix and (right) a horizontal cut.

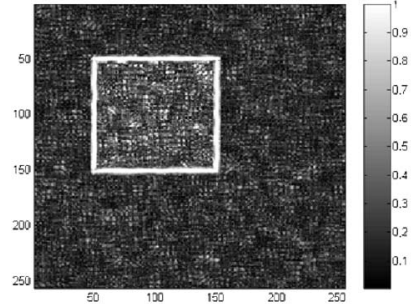

(a)

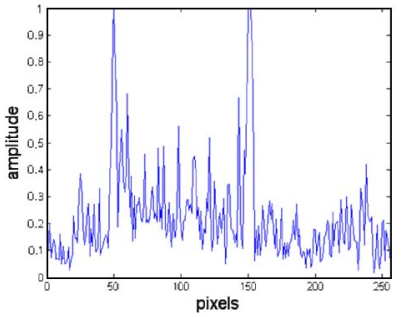

(c)

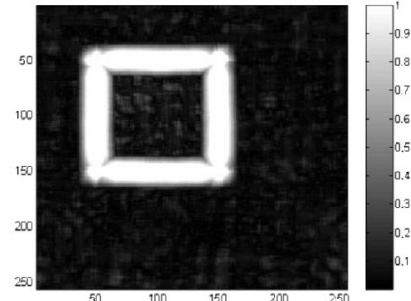

(b)

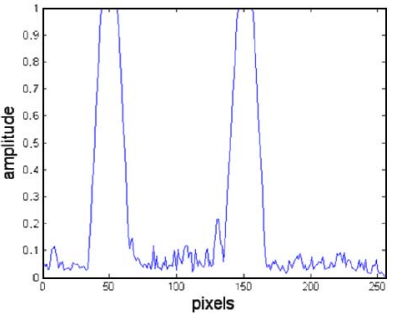

(d)
Fig. 8. Pointwise maxima of the wavelet coefficients at two scales. (a) Interscale pointwise maxima at the third scale of the SWT. (b) Interscale pointwise maxima at the fifth scale of the SWT. (c) Horizontal cut of (a). (d) Horizontal cut of (b).

\section{B. Detailed Example of an Operation in a Simulated Image}

A detailed example of the application of the algorithm proposed for edge enhancement in a simple simulated image is shown hereafter. The image is a speckle matrix $n(\tau, \eta)$ in which a square with an increased intensity has been embedded (see Fig. 7). The intensity in the square has been augmented through multiplication with a constant value $m$

$$
I(\tau, \eta)=m \cdot n(\tau, \eta), \quad \text { with } m \in \mathbb{R} .
$$

In the example treated in this section, $m=5$. The speckle amplitude matrix has been generated as the modulus of a matrix whose both real and imaginary parts are random elements, normally distributed with zero mean and variance equal to 0.5 . A horizontal cut of the image has been included, which is partially superimposed. From its observation, the inefficiency of performing edge enhancement by means of gradient evaluation can be deduced. Furthermore, the multiplicative nature of the speckle is appreciable: The intensity of the noiselike pattern increases with the intensity of the information.

At each iteration, the pointwise maxima of the absolute value of the normalized subbands are evaluated. Two of these intermediate components are shown in Fig. 8. We can observe the effect of the previously discussed tradeoff between resolution and

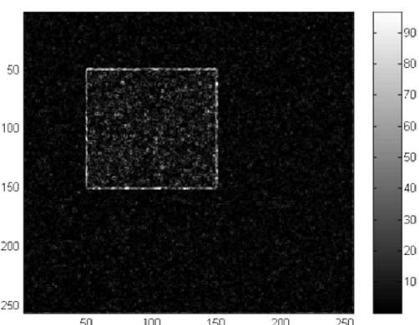

(a)

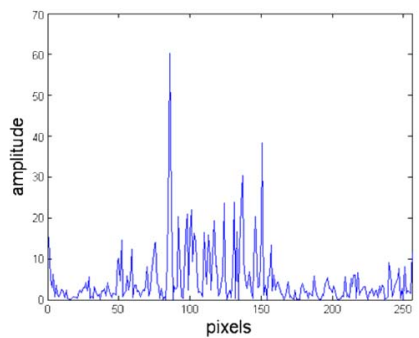

(c)

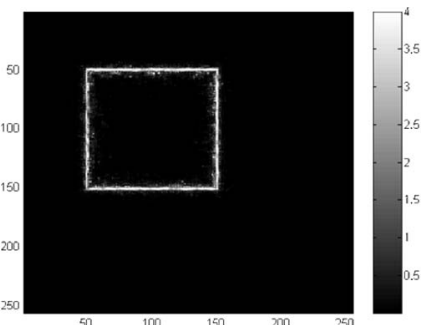

(b)

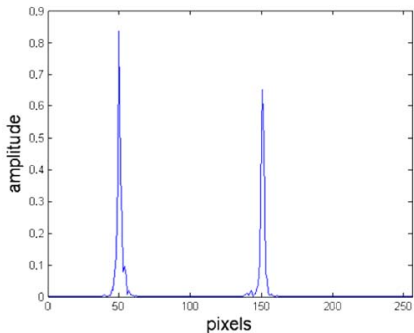

(d)
Fig. 9. Edge enhancement of the simulated image as obtained (a) with the Sobel filter and (b) with the proposed approach. (c) Horizontal cut of (a). (d) Horizontal cut of (b).

signal-to-noise ratio. In both images, the presence of the square contours has been effectively enhanced. Nevertheless, at the fifth scale, the contrast between the edge and the background is noticeably higher than that obtained at the lower scale. On the other way, frontiers appear blurred and thick in the higher scale.

The multiscale pointwise product conciliates these two benefits, aiming to overcome the time-frequency tradeoff that is inherent to wavelet decomposition. Fig. 9 shows the result of the application of the proposed approach on the simulated image in Fig. 7, compared to the result obtained by means of the Sobel filter. The contour of the square appears neatly in the output of the proposed algorithm. The horizontal cut that is superimposed to the output image reflects the enhancement of the contrast achieved between the edge and the surrounding background.

The square shape presented in the previous examples is useful for illustrative purposes, but it may be thought as too simple. Hence, a more complicated shape has been simulated by drawing a coast by means of random $+1 /-1$ displacements along the vertical direction. The results are shown in Fig. 10.

\section{Main Properties of the Algorithm}

This section is devoted to the review of the main properties of the algorithm proposed for automatic edge enhancement in SAR images.

The first consideration concerns simplicity. The proposed technique is simple, and its computational cost is low. It is an iterative process just requiring the following two operations per iteration: the application of a single iteration of the SWT and the evaluation of the pointwise maxima. No previous radiometric calibration is required [29] since this application is not concerned with a precise retrieval of radar cross section values but just with contrast in intensity. Moreover, no prefiltering step is added, and the method is not dependent on the statistics of the input image. 
One of the main interests of the algorithm is that it provides a result directly in the wavelet domain. As a consequence, contrary to conventional filters, it does not require any inversion step, such as in [7], which is usually an awkward process, often introducing artifacts when wavelet coefficients are processed. On the other hand, working on the transformed domain implies considering differential values rather than absolute ones. Hence, the dependence on the overall power of the input image is not so tight and problematic.

Nevertheless, at first sight, the most noticeable effect of the proposed technique is the contrast achieved between edges and background, which is, in fact, the main objective of any edge enhancement algorithm. In order to evaluate the performance of the proposed method, a contrast parameter $C P$ will be considered. It accounts for the contrast obtained in the result between the intensity of the edge and that of the surrounding area as a function of the contrast between the two regions delimiting the edge in the input image. More specifically, the contrast in the result is defined as the difference between the mean value of the intensity of the pixels that correspond to the edge $I_{e}$ and that of the pixels that correspond to the background $I_{b}$, divided by the mean of the background. Similarly, the contrast between the regions in the input image is defined as the difference between the mean intensity of the brightest region at one side of the edge $I_{r 1}$ and that of the other one $I_{r 2}$, divided by the mean of the latter

$$
C P=\left|\frac{\frac{I_{e}-I_{b}}{I_{b}}}{\frac{I_{r 1}-I_{r 2}}{I_{r 2}}}\right| .
$$

The graph in Fig. 11 shows the evolution of the $C P$ parameter for the simulated images as a function of the constant multiplicative value $m$ [see (18)]. It can be deduced that the gain in contrast can be considered as having a linear behavior. The $C P$ parameter cannot be estimated reliably in nonsimulated images. Nevertheless, just as an example, for quantitative comparison purposes, in the simulated situation shown in Fig. 9, the $C P$ that is obtained with the Sobel algorithm after the application of the Lee filter is 1.5 , while the one reached by the proposed technique is 250 .

\section{Application to Coastline Enhancement in SAR Images}

In the framework of this paper, the proposed method has been essentially employed for automatic coastline detection in SAR images. A set of SAR images has been taken into consideration for validation purposes. RADARSAT, ENVISAT, and ERS 1 data with different characteristics (acquisition mode and resolution) have been tested. They correspond to maritime scenarios in which the main objective is to enhance the shoreline.

The interest of performing an automatic extraction of the coastline from remote-sensing data is meaningful. It is sometimes challenging to obtain a precise map of the coastline in particular regions and circumstances with other conventional tools. Moreover, the coastline is subject to a temporal evolution: slow and natural due to erosion, abrupt and natural due to an environmental disaster such as a tsunami, and abrupt and artificial due to a man-made alteration of the coastline. The

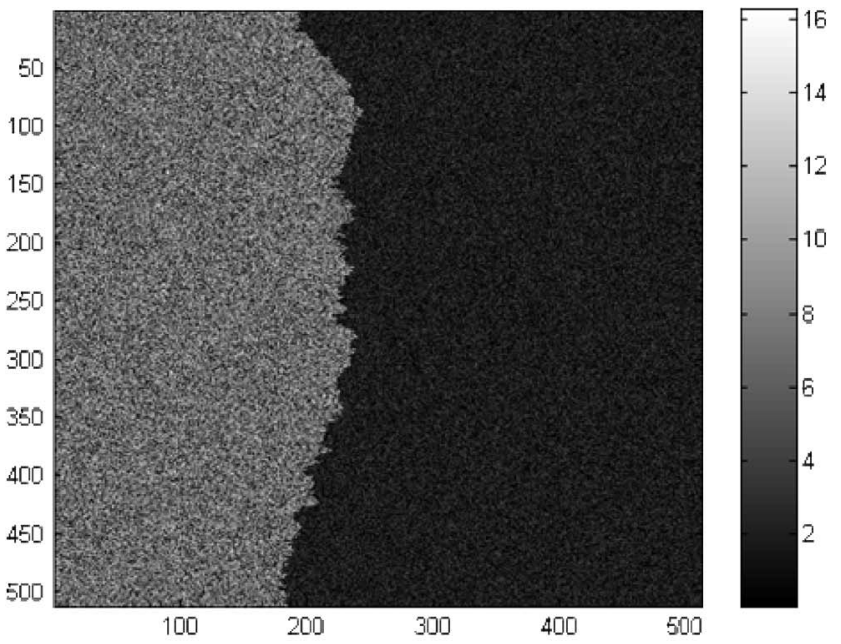

(a)

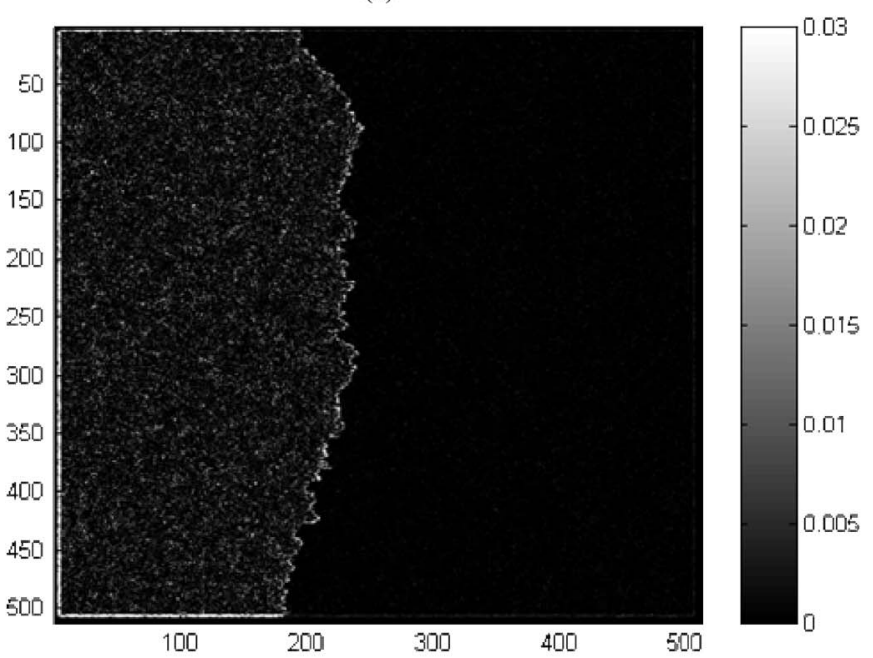

(b)

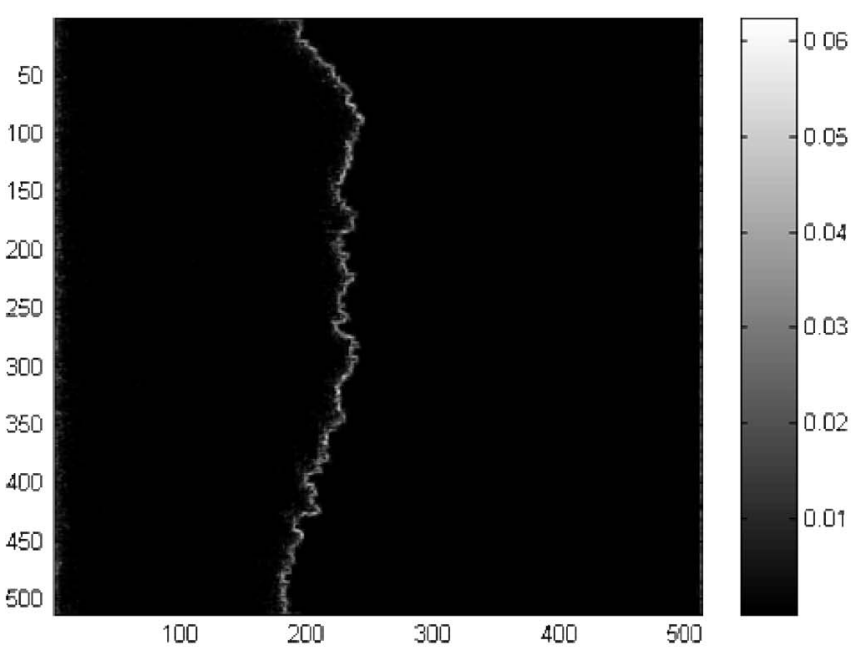

(c)

Fig. 10. Edge enhancement of the simulated image in (a). (b) Result of the application of the Sobel filter. (c) Result of the application of the proposed approach.

monitoring of the coast provides useful information about the behavior of the ocean currents or a wide variety of patterns related to climate change. From an economical point of view, for coastal management and planning, it is crucial to monitor 


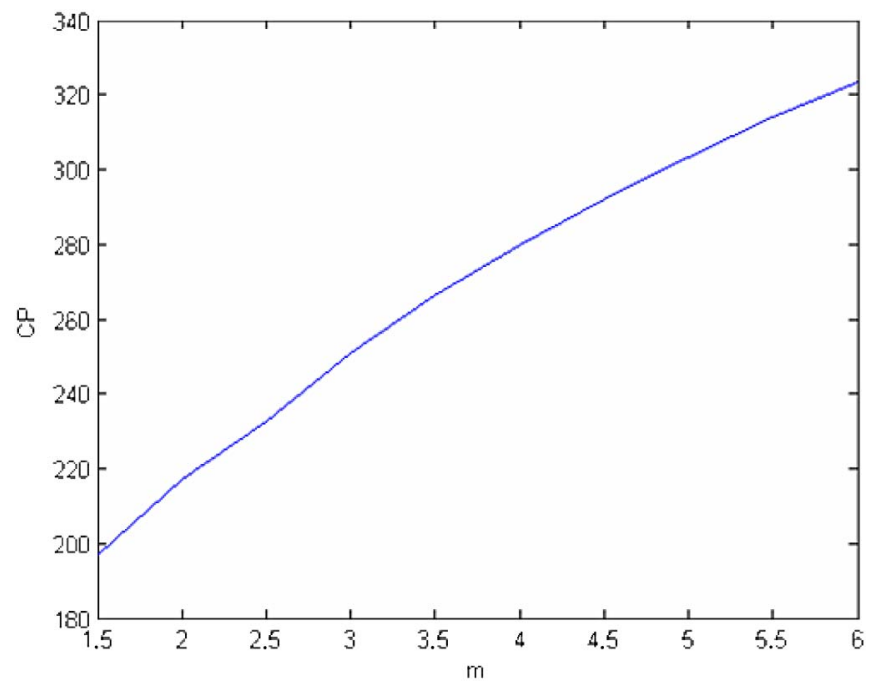

Fig. 11. Contrast enhancement as a function of the input contrast.

the zones that are subject to long-term erosion or to evaluate the affected zones in case of a natural disaster, through the evaluation of the deformations in the coastline. Furthermore, the automatic extraction of the coastline in remote-sensing images is extremely useful as a segmentation tool in other applications. For example, when performing automatic ship detection, a previous land mask step has to be included in any algorithm. It consists of eliminating the land areas, which have to be neglected when looking for vessels. Due to the speckle, automatic land masking in SAR images is an awkward process. Up to now, it is usually performed by using auxiliary external data such as maps available from other sources. Nevertheless, on the one hand, geopositioning of the satellite image has a limited accuracy, and this can lead to image shifts in the azimuth direction, together with a consequent displacement with respect to the land mask. On the other hand, available land masks are of limited accuracy, and there are problems with small islands and coastal man-made constructions. Hence, to overcome this issue, it would be extremely beneficial to perform land mask directly on the remote-sensing image through an efficient technique of segmentation. General difficulties associated with image processing in SAR data have already been overviewed. In the particular framework of the automatic extraction of the coastline, several obstacles have to be considered. The most remarkable one is the diversity of the images. In some images, the energy that is backscattered by the land is higher than that returned by the sea surface, and thus, land areas tend to appear brighter than the ocean ones. Nevertheless, this situation is reverted in other cases, even between two acquisitions that belong to the same area of observation. Moreover, nonstationarity, both in time and space, of the multiple involved processes often results in images that exhibit, at different areas, opposite situations: a sea surface that is brighter than the inland zone and vice versa. Even in some situations, there is absolutely no contrast between regions that are to be segmented. As a consequence, a detection that is exclusively based on the intensity value is not viable.

The test set is composed of neat situations (homogeneous, with a considerable contrast between the land and the sea, together with the awkward ones, and heterogeneous, with the land and sea areas almost undistinguishable). For comparison purposes, the same examples have been processed with the application of a Sobel filter after smoothing with a Lee filter with a window size of $11 \times 11$ pixels and after applying the homomorphic transform in order to transform the multiplicative nature of the speckle noise in the additive one. This window size has been chosen since it is empirically proven to be well suited for the characteristics of the data considered. Some illustrative results are shown in Fig. 12.

The first observation concerns computational efficiency. Applying a Lee filter prior to a Sobel method is drastically more time consuming than performing edge enhancement with the proposed technique. For example, for a $1024 \times 1024$ input matrix, the first option lasts four times more than the second one.

On the other hand, even after filtering with the Lee method, the Sobel operator, which is very sensitive to heterogeneities, is affected by the speckle. Hence, edges appear even in homogeneous sea areas. In contrast, if the regions to be segmented are sufficiently homogeneous, the proposed multiscale technique enhances efficiently the frontier between them, with a large contrast between the coastline and the background: The background is considered to be composed of both water and inland areas. Moreover, the shoreline appears sufficiently thin because the proposed algorithm degrades the resolution just slightly when compared to other methods of smoothing. In fact, as shown in Section IV, the combination of the scales preserves the good localization capabilities of the finest scales, and since the mother wavelet selected for this application is the Haar function with two coefficients, the technique is affected by the loss of the resolution due to the averaging of only two pixels. Additionally, in similar homogeneous scenarios, the operation of the algorithm is benefited if the contrast between the regions is high, since the edge is more pronounced and easily detectable.

In nonhomogeneous scenes, the proposed technique is sensitive to edges produced by structures other than the coastline such as, for example, patterns that are due to topography, rivers, or urban areas. Nevertheless, it is worth noting that these enhanced edges are to be understood as effective ones and not as artifacts that are introduced by the algorithm.

\section{Edge Detection With the Geodesic Active Contour}

\section{A. Theoretical Principles}

Once the transition estimation has been completed, a final decision step is required to build an unsupervised edge detection algorithm. It consists of the segmentation of the input image in the following two classes: edges and no edges. The following two options are to be considered: binarization through thresholding and use of an active contour. The straightforward strategy for segmentation is gradient binarization through thresholding. This option is usually not robust for several reasons. Mainly, the threshold is difficult to define automatically. Furthermore, noise and artifacts may appear, and contours may not be closed. As an alternative to thresholding, the use of active contours, even if more computationally costly, 


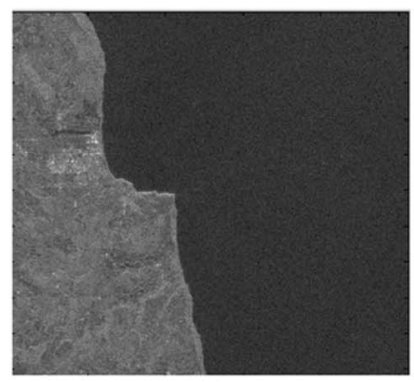

(a)

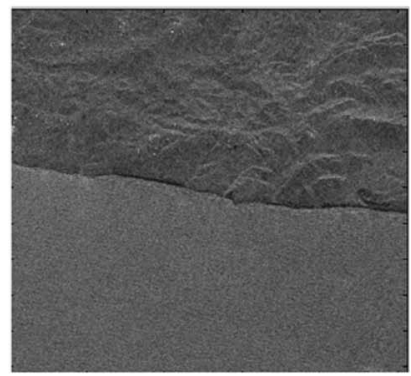

(d)

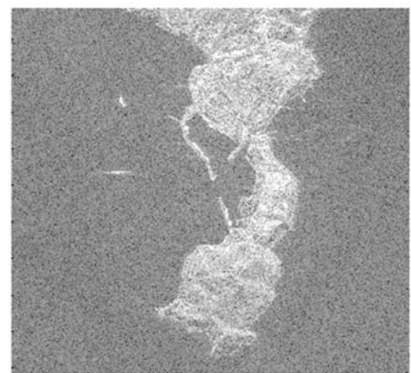

(g)

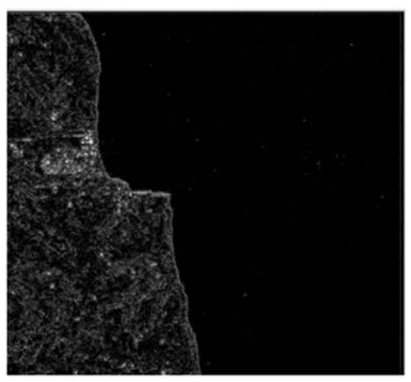

(b)

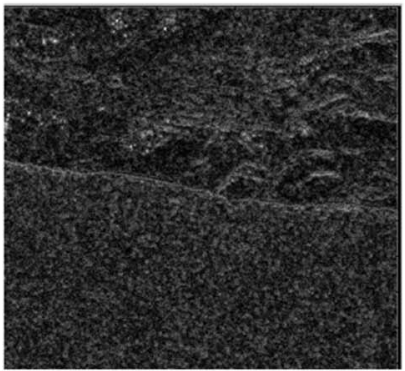

(e)

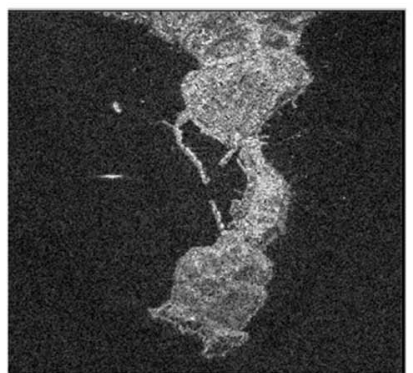

(h)

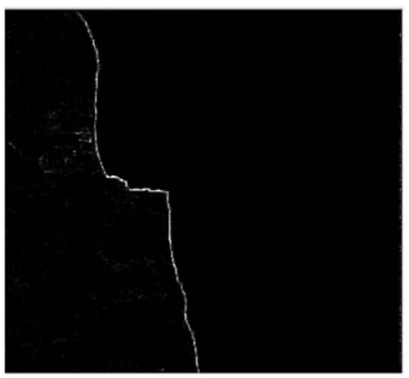

(c)

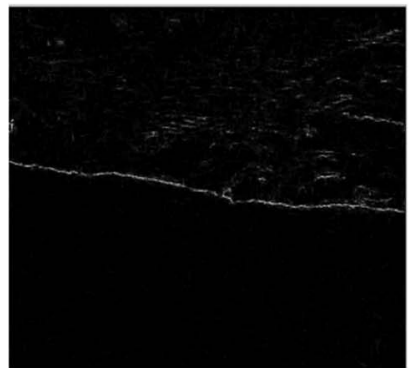

(f)

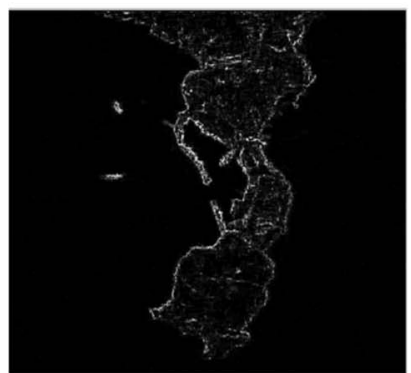

(i)

Fig. 12. Examples of the operation of the multiscale algorithm proposed for edge enhancement in SAR images, compared to Lee + Sobel edge enhancement. (Left column) Input SAR images. (a) ENVISAT ASAR (IM mode) acquired on July 24, 2003. (d) ERS1 (PRI image) acquired on July 19, 1993. (g) RADARSAT 1 acquired on September 26, 2003. (Middle column) Result of the application of the Sobel edge enhancement algorithm after Lee filtering with a window size of $11 \times 11$ pixels. (Right column) Result of the proposed algorithm for edge enhancement.

is preferred. Essentially, a geodesic active contour or snake consists of forcing the evolution of a close curve toward the points of high gradients. More specifically, let the geodesic length $(G L)$ be defined as $G L=\oint g(\nabla x) d s$, where $g$ is a function of $\nabla x$, which is the gradient estimated through the edge enhancement algorithm

$$
g(\nabla x)=\frac{1}{1+\|\nabla x\|^{p}}, \quad \text { with } p \in \mathbb{R} .
$$

In the scope of the examples studied in this paper, $p$ is set to one. Hence, the objective of the snake is to find the curve $C(s)$ such that $G L$ is minimum. This objective function can be minimized by the steepest descent. If we consider $C$ to be a function of time $t$, the Euler-Lagrange equations yield the curve evolution equation [30]

$$
\frac{\partial C}{\partial t}=g \kappa \vec{N}-\langle\nabla g, \vec{N}\rangle \vec{N}
$$

where $\kappa$ is the Euclidean curvature, $\vec{N}$ is the unit inward normal, $\langle.,$.$\rangle stands for the scalar product, and \nabla$ stands for the gradient operator. Nevertheless, from the point of view of practical implementation, it is worth using level-set methods.
In that case, instead of evolving the 1-D curve $C$, we evolve a 2-D surface $u$. $C$ is then the zero level set of $u$, and $u$ is said to be an implicit representation of $C$. It is shown in [31] that the evolution of $u$ can be expressed as

$$
\frac{\partial u}{\partial t}=g(\kappa+c)\|\nabla u\|+\left\langle\nabla g, \frac{\nabla u}{\|\nabla u\|}\right\rangle\|\nabla u\|
$$

where $c$ is a constant erosion parameter and $\kappa$ is defined as

$$
\kappa=\operatorname{div}\left(\frac{\nabla u}{\|\nabla u\|}\right)
$$

where div is the divergence operation. Hence, the minimization is done by initially setting a default surface $u_{0}$ (i.e., $u$ at $t=0$ ) and then by actualizing it iteration after iteration (each iteration represents a differential of time $d t$ ), according to

$$
u_{t}(x, y)=u_{t-1}(x, y)+d t \cdot \beta
$$

where

$$
\beta=g(x, y) \cdot(\kappa+c)\|\nabla u\|+\left\langle\nabla g, \frac{\nabla u}{\|\nabla u\|}\right\rangle\|\nabla u\| .
$$




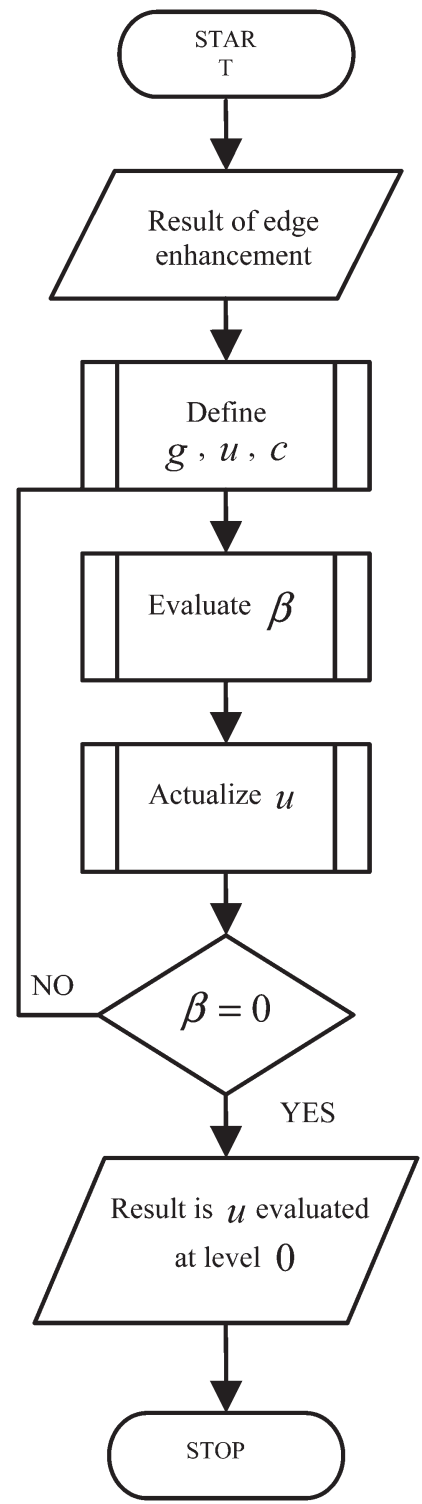

Fig. 13. Flowchart of the geodesic active contour.

Fig. 13 summarizes the different steps for the implementation of the geodesic active contour.

In this paper, the default surface $u_{0}$ is calculated as the distance of every point of the surface to the frame of the image. Hence, the curve that is constituted by $u_{0}$ evaluated at level 0 is the external contour of the input image. It must be noted that this default initial contour often requires a large number of iterations before convergence to the targeted edge. Therefore, in order to save the computational cost, the initial contour should be as close as possible to the final result. An easy way to handle this issue is to consider the highest scales that provide a very rough estimation of the contour. Taking this rough contour as the initial contour of the geodesic active contour considerably enhances the computational performance of the algorithm.

\section{B. Results}

1) Definition of the Quality Parameters: The estimation of the goodness of the segmentation is difficult and application dependent. Probably due to this fact, it is worth noting here the lack in the literature of a consensus of the quality parameters concerning edge detection. As a consequence, the comparison between different techniques usually relies exclusively on mere appreciation factors. Nonetheless, in order to provide a quantitative estimation of the goodness of the approach, three parameters are proposed and defined in this section.

The first parameter concerns the capability of the localization of the algorithm, which is the closeness of the detected edge to the location of the real edge in the input image. Mathematically, there are different parameters measuring the distance between curves. The most extended one is the Hausdorff distance. The Hausdorff distance $H(A, B)$ between two sets of points on two curves $A$ and $B$ is the maximum over each element $a$ of $A$ of the minimum over each element $b$ of $B$ of the distance $d$, according to the underlying metric in the plane, from $a$ to $b$. More concisely

$$
H(A, B)=\max _{a \in A}\left(\min _{b \in B}(d(a, b))\right) .
$$

By taking into account the characteristics of the application, the objective here is to provide a measure of the error, which is the distance between the real and retrieved edges. Hence, an appropriate measure of the error in a discrete context can be defined as

$$
\text { error }=\frac{1}{\operatorname{card}(A)} \sum_{a \in A} \min _{b \in B}(d(a, b)) .
$$

From a practical point of view, the evaluation of this parameter has been implemented as follows. For every single point of the longest curve, its distance to the closest point in the other curve is computed. Then, the mean value of this set of distances constitutes the error. This error makes sense only if the edge is effectively detected but misplaced. Two additional rates are estimated in order to account for misdetections.

Additionally, the following two values are estimated: the probability of false positives $p_{\mathrm{FP}}$ and the probability of false negatives $p_{\mathrm{FN}}$. The probability of false negatives measures the quantity of elements that belong to class edge and that are misclassified as no edge, and similarly, the probability of false positives reflects the quantity of individuals that are classified as edge and that are contrarily being part of no edge. Let us define the following four classes: $E_{\text {input }}$ confirmed edges in the input image, $E_{\text {output }}$ detected edges (i.e., "edges in the output image"), $N E_{\text {input }}$ confirmed no edges in the input image, and $N E_{\text {output }}$ detected no edges (i.e., no edges in the output image). With this

$$
\begin{aligned}
& p_{\mathrm{FP}}=\frac{\operatorname{card}\left\{E_{\text {output }} \cap N E_{\text {input }}\right\}}{\operatorname{card}\left\{E_{\text {output }}\right\}} \\
& p_{\mathrm{FN}}=\frac{\operatorname{card}\left\{E_{\text {input }} \cap N E_{\text {output }}\right\}}{\operatorname{card}\left\{E_{\text {input }}\right\}} .
\end{aligned}
$$

It is worth noting that these values make sense only if an existing edge is not detected or if the algorithm finds nonexisting edges. However, the rates $p_{\mathrm{FP}}$ and $p_{\mathrm{FN}}$ are to be understood as 


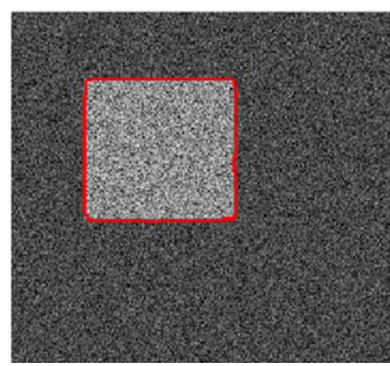

(a)

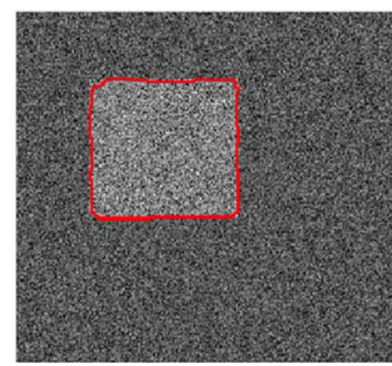

(b)

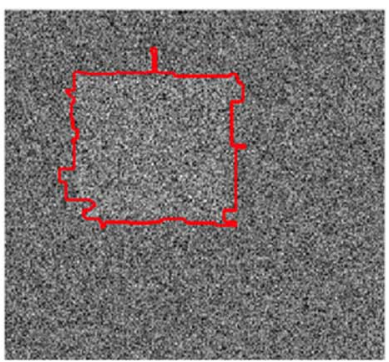

(c)

Fig. 14. Examples of the detection of the overall chain of coastline extraction on simulated images. (a) $m=2.5$, error $=0.1125, p_{\mathrm{FP}}=0$, and $p_{\mathrm{FN}}=0$. (b) $m=1.5$, error $=0.25, p_{\mathrm{FP}}=0$, and $p_{\mathrm{FN}}=0$. (c) $m=1.2$, error $=1.1625, p_{\mathrm{FP}}=0.06$, and $p_{\mathrm{FN}}=0.1$.

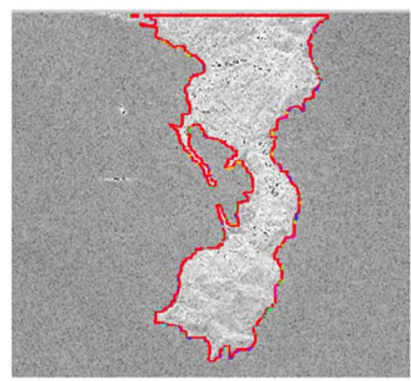

(a)

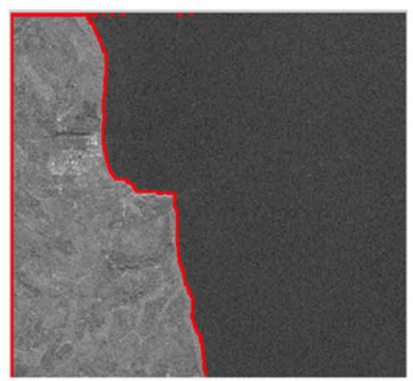

(b)

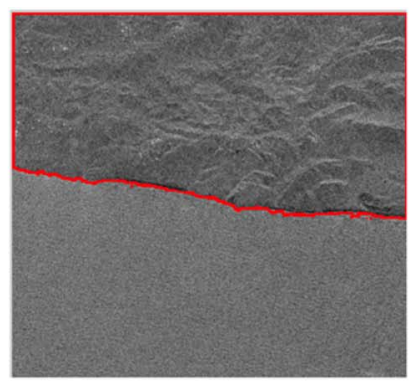

(c)

Fig. 15. Examples of the detection of the overall chain of coastline extraction on SAR images.

complementary to the error parameter defined previously: If an existing edge is detected but misplaced, $p_{\mathrm{FP}}$ is considered null. These quality parameters can be estimated precisely in simple simulated images, but their evaluation is usually unfeasible in real images with no available ground truth.

2) Analysis of the Results: This section presents the examples of the results of the whole chain of edge detection constituted by the edge enhancement algorithm before the application of a geodesic active contour. An example of the operation on simulated images will be first considered. The images are synthesized, as previously specified, as speckle matrices with squares embedded. Several examples are shown in Fig. 14. The corresponding parameters as well as the evaluated errors are included in the figure. Fig. 15 shows three examples of coast detection by means of the method proposed in this paper in the SAR images introduced in Fig. 12. Since the quality parameters discussed in the previous section are not possible to evaluate in real scenarios, a good way to have a visual evaluation of the goodness of the method is to superimpose the shoreline extracted to the input SAR image.

The observation of the results obtained in SAR images confirms a good match of the detected coastline to the shoreline that is visually inferred. It is worth noting that the red line has been highlighted for representation purposes, but the result is one pixel wide. Moreover, the correct performance of the method is also observed despite the diversity of scenarios.

\section{CONCLUSION}

In this paper, a multiscale algorithm for the unsupervised extraction of the most significant edges has been presented. It has been designed specifically to deal with speckled SAR images. The method proposes a robust edge enhancement directly in the wavelet transformed domain, followed by a decision step based on the application of a geodesic active contour algorithm. The edge enhancement phase has been proven to be critical in heterogeneous SAR images, and the original method proposed in this paper constitutes a good solution that is used to deal with this type of data. It does not require any type of prefiltering of data, and it is independent of the statistics of the input image. The adaptation capability of the method to very diverse scenarios with no need of a priori knowledge or settings is a useful feature in view of its integration in an unsupervised chain.

After testing the performance of the technique proposed in the simulated images, it has been applied to real data in the framework of coastline extraction from SAR images. It has been proven to be robust and effective for this application. The extracted coastline, which is an entirely connected line of one pixel wide, matches accurately the targeted frontier. Moreover, this method could be effectively used for segmentation purposes in SAR data.

\section{ACKNOWLEDGMENT}

The authors would like to thank the European Space Agency for providing the SAR images.

\section{REFERENCES}

[1] W. K. Pratt, Digital Image Processing. New York: Wiley, 1978.

[2] J. M. S. Prewitt, "Object enhancement and extraction," in Picture Processing and Psychopictorics, B. S. Lipkin and A. Rosenfeld, Eds. New York: Academic, 1970, pp. 75-149. 
[3] J. W. Goodman, Speckle Phenomena in Optics: Theory and Applications. Englewood, CO: Roberts \& Company, 2007.

[4] B. M. Sadler and A. Swami, "Analysis of multiscale products for step detection and estimation," IEEE Trans. Inf. Theory, vol. 45, no. 3, pp. 10431051, Apr. 1999.

[5] G. Cuartero, V. Meas-Yedid, V. Galy, U. Nehrbass, and J.-C. OlivoMarine, "Three dimensional spot detection by multiscale analysis," in Proc. Int. Conf. Image Process., Oct. 7-10, 2001, vol. 1, pp. 317-320.

[6] J. Kuo and K.-S. Chen, "The application of wavelets correlator for ship wake detection in SAR images," IEEE Trans. Geosci. Remote Sens., vol. 41, no. 6, pt. 2, pp. 1506-1511, Jun. 2003.

[7] Y. Xu, J. B. Weaver, D. M. Healy, and J. Lu, "Wavelet transform domain filters: A spatially selective noise filtration technique," IEEE Trans. Image Process., vol. 3, no. 6, pp. 747-758, Nov. 1994.

[8] I. Melgar, J. Fombellida, J. Gomez, and J. Seijas, "Edge detection algorithms in intensity SAR images: Empirical comparison of performances and proposed improvements," in Proc. WAC, Sep. 2008, pp. 1-7.

[9] R. Touzi, A. Lopès, and P. Bousquet, "A statistical and geometrical edge detector for SAR images," IEEE Trans. Geosci. Remote Sens., vol. 26, no. 6, pp. 764-773, Nov. 1988.

[10] R. G. White and C. J. Oliver, "Change detection in SAR imagery," in Proc. IEEE Int. Radar Conf., May 1990, pp. 217-222.

[11] C. J. Oliver, D. Blacknell, and R. G. White, "Optimum edge detection in SAR," Proc. Inst. Elect. Eng.-Radar, Sonar, Navigat., vol. 143, no. 1, pp. 31-40, Feb. 1996.

[12] R. Fjortoft, A. Lopes, P. Marthon, and E. Cubero-Castan, "An optimal multiedge detector for SAR image segmentation," IEEE Trans. Geosci. Remote Sens., vol. 36, no. 3, pp. 793-802, May 1998.

[13] E. Romaneessen, A. Niedermeier, N. Winkel, and S. Lehner, "Improved bottom topography in the Elbe estuary using wavelet and active contour methods on SAR images," in Proc. IGARSS, Jul. 1999, vol. 3, pp. 1674-1676.

[14] S. Madchakham, P. Thitimajshima, and Y. Ragsanseri, "Edge detection in speckled SAR images using wavelet decomposition," in Proc. ACRS, Nov. 2001, vol. 2, pp. 1307-1310.

[15] C. Oliver and S. Quegan, Understanding Synthetic Aperture Radar Images. Norwood, MA: Artech House, 1998.

[16] J. C. Curlander and R. N. McDonough, Synthetic Aperture Radar: Systems and Signal Processing. Hoboken, NJ: Wiley, 1991.

[17] R. Bamler and P. Hartl, "Synthetic aperture radar interferometry," Inverse Probl., vol. 14, no. 4, pp. R1-R54, Aug. 1998.

[18] S. R. Cloude and K. P. Papathanassiou, "Polarimetric SAR interferometry," IEEE Trans. Geosci. Remote Sens., vol. 36, no. 5, pp. 1551-1565, Sep. 1998.

[19] K. J. Langenberg, M. Brandfass, K. Mayer, T. Kreutter, A. Brüll, P. Felinger, and D. Huo, "Principles of microwave imaging and inverse scattering," EARSeL Adv. Remote Sens., vol. 2, no. 1, pp. 163-186, Jan. 1993.

[20] F. Ulaby, R. Moore, and A. Fung, Microwave Remote Sensing. Active and Passive. Norwood, MA: Artech House, 1982.

[21] J. W. Goodman, "Some fundamental properties of speckle," J. Opt. Soc. Amer, vol. 66, no. 11, pp. 1145-1149, Nov. 1976

[22] J. S. Lee, "Refined filtering of image noise using local statistics," Comput. Vis., Graph., Image Process., vol. 15, no. 4, pp. 380-389, Apr. 1981.

[23] D. T. Kuan, A. A. Sawchuck, T. C. Strand, and P. Chavel, "Adaptive restoration of images with speckle," IEEE Trans. Acoust., Speech, Signal Process., vol. ASSP-35, no. 3, pp. 373-383, Mar. 1987.

[24] A. N. Evans, "A gamma filter for multi-look synthetic aperture radar images," in Proc. ISSPA, 1996, pp. 829-832.

[25] R. Touzi, "A review of speckle filtering in the context of estimation theory," IEEE Trans. Geosci. Remote Sens., vol. 40, no. 11, pp. 23922404, Nov. 2002.

[26] F. T. Ulaby and C. Elachi, Radar Polarimetry for Geoscience Applications. Norwood, MA: Artech House, 1990.

[27] S. Mallat, A Wavelet Tour of Signal Processing. New York: Academic, 1999.

[28] H. Arsenault and G. April, "Properties of speckle integrated with a finite aperture and logarithmically transformed," J. Opt. Soc. Amer., vol. 66, no. 11, pp. 1160-1163, Nov. 1976.

[29] A. Freeman, "SAR calibration: An overview," IEEE Trans. Geosci. Remote Sens., vol. 30, no. 6, pp. 1107-1121, Nov. 1992.

[30] V. Caselles, R. Kimmel, and G. Sapiro, "Geodesic active contours," Int. J. Comput. Vis., vol. 22, no. 1, pp. 61-79, Feb. 1997.

[31] Y. G. Chen, Y. Giga, and S. Goto, "Uniqueness and existence of viscosity solutions of generalized mean curvature flow equations," J. Differ. Geom., vol. 33, no. 3, pp. 749-786, 1991.

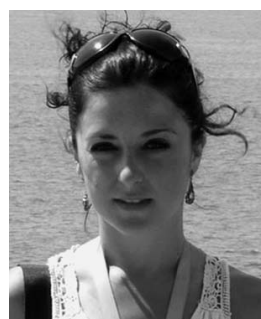

Mariví Tello Alonso (S'03) was born in Barcelona, Spain. She received the degree in telecommunications engineering from the Universitat Politècnica de Catalunya, Barcelona, in 2003, where she is currently working toward the Ph.D. degree in the Remote Sensing Laboratory, Department of Signal Theory and Communications, working on multiscale postprocessing techniques for the unsupervised interpretation of synthetic aperture radar images in the scope of maritime applications such as vessel monitoring, coastline extraction, and oil spill detection.

Since January 2010, she has been with the Institut Català de Ciències del Clima (IC.3). She is involved in remote-sensing applications that are related to climate change. Her research interest is fundamental signal processing applied to remote-sensing data.

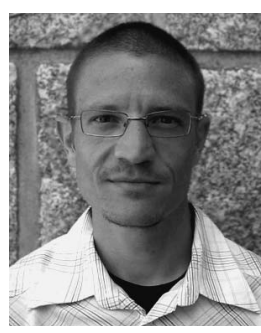

Carlos López-Martínez (S'96-M'04) received the M.Sc. degree in electrical engineering and the Ph.D. degree from the Universitat Politècnica de Catalunya (UPC), Barcelona, Spain, in 1999 and 2003, respectively.

In 1999, he joined the Department of Signal Theory and Communications, UPC, where he worked on his Ph.D. dissertation, which was focused on multidimensional speckle noise modeling and reduction. From October 2000 to March 2002, he was with the Frequency and Radar Systems Department (HR), German Aerospace Center (DLR), Oberpfaffenhofen, Germany. From June 2003 to December 2005, he was with the Image and Remote Sensing Group-SAPHIR Team, Institute of Electronics and Telecommunications of Rennes (IETR - CNRS UMR 6164), Rennes, France. Since January 2006, he has been a Ramón-y-Cajal Fellow with the Remote Sensing Laboratory, Department of Signal Theory and Communications, UPC, where he develops research and teaching activities. His research interests include synthetic aperture radar (SAR) and multidimensional SAR, radar polarimetry, digital signal processing, estimation theory, and harmonic analysis. He has served as an Invited Associate Editor of the EURASIP Journal on Advances in Signal Processing.

Dr. López-Martínez was the recipient of the Student Prize Paper Award at the EUSAR 2002 Conference, Cologne, Germany. He has also served as Chair and Cochair at the IGARSS, EUSAR, and PolInSAR conferences.

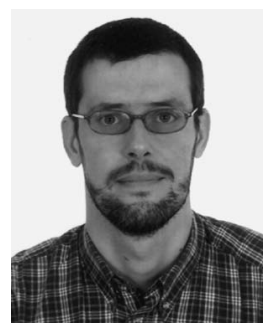

Jordi J. Mallorquí (S'93-M'96) was born in Tarragona, Spain, in 1966. He received the Ingeniero and Doctor Ingeniero degrees in telecommunications engineering from the Universitat Politècnica de Catalunya (UPC), Barcelona, Spain, in 1990 and 1995, respectively, where his Ph.D. dissertation was on microwave tomography for biomedical applications.

Since 1993, he has been with the School of Telecommunications Engineering of Barcelona, UPC, first as an Assistant Professor and then, since 1997, as an Associate Professor. His teaching activity involves microwaves, radionavigation systems, and remote sensing. He spent a sabbatical year with the Jet Propulsion Laboratory, Pasadena, CA, in 1999, working on interferometric airborne synthetic aperture radar (SAR) calibration algorithms. He is currently working on the application of SAR interferometry to terraindeformation monitoring with orbital, airborne, and ground data; vessel detection and classification from SAR images; and 3-D electromagnetic (EM) simulation of SAR systems. He also collaborates in the design and construction of a ground-based SAR interferometer for landslide control. Finally, he is currently developing the hardware and software of a bistatic opportunistic SAR for interferometric applications using ERS, ENVISAT, and TerraSAR-X as sensors of opportunity. He has published more than 80 papers on microwave tomography, EM numerical simulation, and SAR processing, interferometry, and differential interferometry in refereed journals and international symposia. 


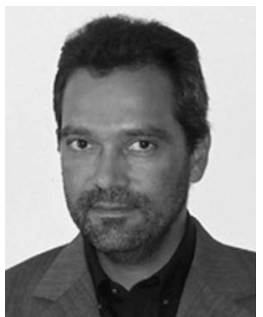

Philippe Salembier (M'96-SM'09) received the B.Sc. degree from the Ecole Polytechnique, Paris, France, in 1983, and the M.Sc. degree from the Ecole Nationale Supérieure des Télécommunications, Paris, in 1985. He received the Ph.D. degree from the Swiss Federal Institute of Technology (EPFL), Lausanne, Switzerland, in 1991.

From 1985 to 1989 , he was with the Laboratoires d'Electronique Philips, Limeil-Brevannes, France, working in the fields of digital communications and signal processing for HDTV. In 1989, he joined the Signal Processing Laboratory, EPFL, working on image processing. He was a Postdoctoral Fellow with the Harvard Robotics Laboratory, Cambridge, MA, in 1991. At the end of 1991, after a stay at the Harvard Robotics Laboratory, he joined the Universitat Politècnica de Catalunya (UPC), Barcelona, Spain, where he is currently a Professor, lecturing on the area of digital signal and image processing. His current research interests include image and sequence coding, compression and indexing, segmentation, video sequence analysis, mathematical morphology, level sets, and nonlinear filtering. In terms of standardization activities, he has been particularly involved in the definition of the MPEG-7 standard ("Multimedia Content Description Interface") as the Chair of the "Multimedia Description Scheme" group between 1999 and 2001. He served as an Area Editor of the Journal of Visual Communication and Image Representation from 1995 to 1998. He was the Coeditor-in-Chief of Signal Processing between 2001 and 2002. He is currently an Associate Editor of the EURASIP Journal on Image and Video Processing.

Dr. Salembier served as an AdCom Officer of the European Association for Signal Processing (EURASIP) from 1994 to 1999 . He was a member of the Image and Multidimensional Signal Processing Technical Committee of the IEEE Signal Processing Society between 2000 and 2006 and was the Technical Chair (with Prof. Ed. Delp) of the IEEE International Conference on Image Processing (ICIP'2003) organized in Barcelona. He served as an Associate Editor of the IEEE TRANSACTIONS ON IMAGE PROCESSING between 2002 and 2008 and of the IEEE Signal Processing LetTers between 2005 and 2008. 